\title{
Linear spatio-temporal instability analysis of ice growth under a falling water film
}

\author{
JUN $\mathrm{HU}^{1} \dagger$, BING-HONG ZHOU ${ }^{2}$, YI-HONG HANG ${ }^{1}$, \\ QIU-SHENG LIU ${ }^{3}$ AND SHU-DAO ZHANG \\ ${ }^{1}$ Institute of Applied Physics and Computational Mathematics, Beijing 100088, China \\ ${ }^{2}$ Center for Space Science and Applied Research, Chinese Academy of Sciences, Beijing 100190, China \\ ${ }^{3}$ Key Laboratory of Microgravity, Institute of Mechanics, Chinese Academy of Sciences, \\ Beijing 100080, China
}

(Received 18 May 2009; revised 13 January 2010; accepted 14 January 2010)

A linear spatio-temporal stability analysis is conducted for the ice growth under a falling water film along an inclined ice plane. The full system of linear stability equations is solved by using the Chebyshev collocation method. By plotting the boundary curve between the linear absolute and convective instabilities (AI/CI) of the ice mode in the parameter plane of the Reynolds number and incline angle, it is found that the linear absolute instability exists and occurs above a minimum Reynolds number and below a maximum inclined angle. Furthermore, by plotting the critical Reynolds number curves with respect to the inclined angle for the downstream and upstream branches, the convectively unstable region is determined and divided into three parts, one of which has both downstream and upstream convectively unstable wavepackets and the other two have only downstream or upstream convectively unstable wavepacket. Finally, the effect of the Stefan number and the thickness of the ice layer on the AI/CI boundary curve is investigated.

\section{Introduction}

The effect of ice formation under a gravity-driven water film is of great practical importance in aircraft icing and shallow drainage conduits used in diverse engineering structures subject to moisture collection. In order to study ice growth in a pipe, Gilpin (1979) performed a series of experiments and found that for the entire range of the Reynolds numbers obtainable in the experiment (up to 14000), there exists a final steady-state ice profile with a cyclic variation in height along the pipe. Interestingly, he observed that the ice-band structure forms in the following way (Shapiro 2004). Initially, ice grows uniformly until some height is gained, afterwards a sharp expansion of the flow passage forms near the exit of the cooled section; moreover, this expansion migrates upstream; as the first expansion migrates upstream, another ice growth may form near the pipe exit and propagates upstream in its turn. From the absolute and convective instability point of view, the development of the ice-band structure shows that the ice growth is convectively unstable and the unstable wavepacket spreads upstream.

Since Kapitza \& Kapitza (1949) experimentally studied the isothermal liquid film on an inclined plane, the mechanisms of the film instability have been extensively

$\dagger$ Email address for correspondence: hu_jun@iapcm.ac.cn 
studied by theoretical linear and nonlinear approaches. Benjamin (1957) and Yih (1963) first performed a linear stability analysis of isothermal falling films and identified the surface wave instability through a long-wave asymptotic approach. DeBruin (1974), Floryan, Davis \& Kelly (1987) and Woods \& Lin (1996) revealed the short shear instability (Tollmien-Schlichting wave) occurring at a slightly inclined angle $\left(<1^{\circ}\right)$. Naturally, if the water film flows on a flat ice layer with a finite thickness, the traditional film model produces an excellent starting point for the analysis of the icing instability. Such a generalization is first adopted by Shapiro \& Timoshin (2006) for the stability study of ice growth. They first derived an analytic solution to the stability problem in the long-wave limit, which shows that the presence of the ice layer generates an additional wave mode, named ice mode by them. Furthermore, by using this long-wave solution as an initial guess, they found the ice mode in the numerical solution of the complete Orr-Sommerfeld problem and investigated its stability boundary behaviour numerically for a wide range of problem parameters, especially giving out fruitful information about the variation of the critical parameters with respect to different inclined angles, Stefan numbers and ice layer thickness.

The linear absolute and convective instabilities for a single-layer isothermal falling film have been studied intensively by Brevdo et al. (1999). They focused on the full linearized Navier-Stokes equations to investigate the characteristics of the absolute and convective instability through the exact Briggs-Bers collision criterion. They explored a large region of the parameter space and pointed out that the one-layer film flow is convectively unstable, which agrees with all done experiments. The absolute and convective instability analysis of ice growth under a falling water film is performed by Shapiro \& Timoshin (2007) through the numerical investigation of the evolution of a spatially localized initial disturbance. They adopted an asymptotic double-deck theory to derive a system of nonlinear evolution equations which is suitable for large Reynolds and Froude numbers, and further used a novel global marching-type scheme to solve the evolution equations and investigate the effects of nonlinearity of the ice instability. They successfully discovered an upstream-propagating instability arising from the interaction of the ice surface with the water film flow. This convectively unstable characteristic agrees with the Gilpin's experiment (Gilpin 1979). So far, the spatio-temporal instability analysis on the full linearized Navier-Stokes equations for the ice growth is still not performed. Because of the potential application of the stability analysis in aircraft icing, especially ice formation corresponding to upstream convective instability or absolute instability will change the aircraft profile greatly from streamlined to non-streamlined, and then the performance of aircraft will reduce violently even leading to catastrophic accidents. Thus, a detailed theoretical study of the linear absolute and convective instabilities of ice growth is necessary, especially investigated within a large parameter region, which is the main focus of this paper.

\section{Formulation}

\subsection{Dimensionless governing equations}

We are concerned with an ice growth from an undercooled thin water film flowing down an inclined plane with angle $\beta$. As sketched in figure 1, there exist two layers: water layer and ice sheet with finite thicknesses. In this model of ice growth, the temperature of the cold solid wall is below freezing point and the temperature of the water surface is above freezing point. We will use subscripts $L$ and $I$ to 


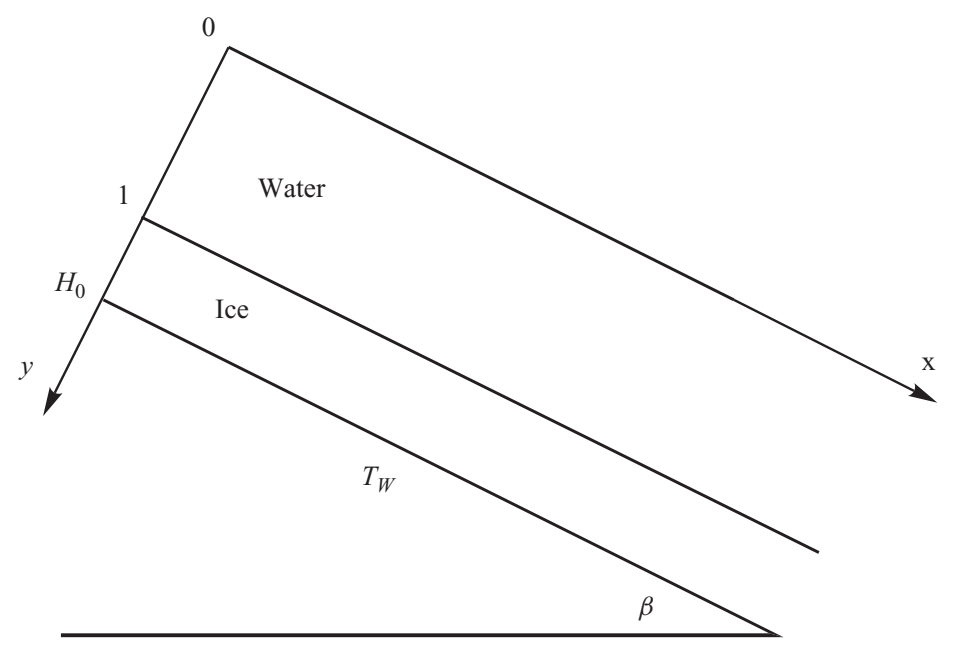

FIGURE 1. Schematic of the water film falling along an ice plane.

refer to the properties of liquid layer and ice sheet, respectively. The system is dimensionalized by the water film thickness $h_{0}$ for length, the free surface undisturbed velocity $u_{0}=g h_{0}^{2} \sin \beta / 2 v_{L}$ for velocity, $\mu_{L} u_{0} / h_{0}$ for pressure and $\Delta T=T_{a}-T_{f}$ for temperature. Here, $\rho_{L}$ denotes the density of water, $\mu_{L}$ and $\nu_{L}$ denote the dynamic and kinematic viscosities of water, respectively, $T_{f}$ denotes the freezing temperature of water, and $T_{a}$ denotes the temperature of the surrounding air, which is assumed to be constant.

Using these characteristic values and the usual notation for velocity and pressure, and neglecting the buoyancy effect, the governing system of equations can be written in non-dimensional form as

$$
\begin{aligned}
\nabla \cdot \boldsymbol{v} & =0, \\
\frac{\mathrm{D} \boldsymbol{v}}{\mathrm{D} t} & =-\frac{1}{\operatorname{Re}} \nabla p+\frac{1}{\operatorname{Re}} \nabla^{2} \boldsymbol{v}+\frac{1}{F^{2}} \boldsymbol{e}_{g}, \\
\frac{\mathrm{D} \theta_{L}}{\mathrm{D} t} & =\frac{1}{\operatorname{Pr} \operatorname{Re}} \nabla^{2} \theta_{L}, \\
\frac{\partial \theta_{I}}{\partial t} & =\frac{\chi_{I L}}{\operatorname{Pr} R e} \nabla^{2} \theta_{I} .
\end{aligned}
$$

Here, $\nabla=\left(\partial_{x}, \partial_{y}\right), \boldsymbol{v}=(u, v)$ and $\boldsymbol{e}_{g}=(\sin \beta,-\cos \beta)$ are vectors of the gradient operator, dimensionless fluid velocity and gravity acceleration unit vector, $p$ is the dimensionless fluid pressure, $\theta_{L}$ and $\theta_{I}$ are the corresponding dimensionless temperatures of the water layer and the ice sheet, respectively. The dimensionless parameters appearing in the above governing equations are Reynolds number, Prandtl number and Froude number and are defined as

$$
R e=\frac{u_{0} h_{0}}{\nu_{L}}, \quad \operatorname{Pr}=\frac{\nu_{L}}{\chi_{L}}, \quad F r=\frac{u_{0}}{\left(g h_{0}\right)^{1 / 2}} .
$$

Also, $\chi_{I L}=\chi_{I} / \chi_{L}$ is the ratio of thermal diffusivities of the ice and the water. 
The dimensionless boundary conditions at the free surface $y=\xi(x, t)$ are

$$
\begin{aligned}
\xi_{t}+u \xi_{x} & =v, \\
\boldsymbol{T} \cdot \boldsymbol{n}_{s}+W e \kappa \boldsymbol{n}_{s} & =0, \\
\theta_{L} & =1,
\end{aligned}
$$

where $\boldsymbol{n}_{s}$ is the unit outward vector normal to the free surface, the stress tensor $\boldsymbol{T}$ and mean curvature $\kappa / 2$ are represented as

$$
\begin{gathered}
\boldsymbol{T}=-p \boldsymbol{I}+\left(\nabla \boldsymbol{v}+(\nabla \boldsymbol{v})^{\mathrm{T}}\right), \\
\kappa=-\nabla \cdot \boldsymbol{n}_{s},
\end{gathered}
$$

and $W e$ is the Weber number defined as

$$
W e=\frac{2 \sigma}{\rho_{L} g h_{0}^{2} \sin \beta}=R e^{-2 / 3}\left(\frac{3}{2} \sin \beta\right)^{-1 / 3} \zeta .
$$

Here, $\sigma$ is the surface tension coefficient, which is assumed to be independent of the temperature, i.e. the thermocapillary effect is neglected. Moreover, $\zeta=\left[3 \rho_{L} \sigma^{3} / g \mu_{L}^{4}\right]^{1 / 3}$ is the surface tension parameter that depends only on media properties.

The dimensionless boundary conditions at the water-ice interface $y=\eta(x, t)$ are

$$
\begin{gathered}
\phi_{I L} \eta_{t}+u \eta_{x}=v, \\
\left(\lambda_{I L} \nabla \theta_{I}-\nabla \theta_{L}\right) \cdot \boldsymbol{n}_{i}=\frac{\operatorname{Pr} R e}{S t}\left[1+\Lambda_{1} \frac{\left(\boldsymbol{v}-\boldsymbol{u}_{s}\right)^{2}-\boldsymbol{u}_{s}^{2}}{2}\right] \boldsymbol{v} \cdot \boldsymbol{n}_{i}+\Lambda_{2}\left(\boldsymbol{v}-\boldsymbol{u}_{s}\right) \cdot \boldsymbol{S} \cdot \boldsymbol{n}_{i}, \\
\theta_{L}=\theta_{I}=0,
\end{gathered}
$$

where $\boldsymbol{S}=\left(\nabla \boldsymbol{v}+(\nabla \boldsymbol{v})^{\mathrm{T}}\right)$ is the deviatoric stress tensor, $\boldsymbol{u}_{s}$ is the interface velocity of phase change, $\boldsymbol{n}_{i}$ is the unit vector normal to the interface that directs from the ice to the water, $\phi_{I L}=1-\rho_{I} / \rho_{L}$ is a positive constant involving only the densities of the ice and and water, $\lambda_{I L}=\lambda_{I} / \lambda_{L}$ is the ratio of thermal conductivities of the ice and the water, and the Stefan number $S t, \Lambda_{1}$ and $\Lambda_{2}$ are defined as

$$
S t=\frac{\left(\rho_{L}-\rho_{I}\right) C p_{L} \Delta T}{\rho_{I} H_{L I}}, \quad \Lambda_{1}=\frac{u_{0}^{2}}{H_{L I}}, \quad \Lambda_{2}=\frac{\mu_{L} u_{0}^{2}}{\lambda_{L} \Delta T} .
$$

Here, $C p_{L}$ is the water-specific heat and $H_{L I}$ is the latent heat, which is defined by the amount of heat required to change a unit mass of solid into liquid at the freezing temperature. We argue that the definition of Stefan number here is different from that of Gilpin (1979) for there is an additional part $\left(\rho_{L}-\rho_{I}\right) / \rho_{I}$.

The boundary condition at the bottom cold wall is only the temperature condition due to no motion of the ice sheet:

$$
\theta_{I}=T_{W}=\text { constant }
$$

Note that the Stefan boundary condition (2.6b) is deduced from the complete form of mass, momentum and energy balance equations (Lin \& Hudman 1996) at the ice-water interface. Furthermore, there exist two nonlinear terms that arise from the work done by the viscous stress and the kinetic energy, respectively. However, for most situations with moderate scale of velocity, the dimensionless parameters in $(2.6 b)$ have

$$
\Lambda_{1} \ll 1, \quad \text { and } \quad \Lambda_{2} \ll \frac{\operatorname{Pr} R e}{S t}
$$


Name

Density

Thermal conductivity

Specific heat

Thermal diffusivity

Dynamic viscosity

Latent heat
Symbol

$\rho$

$\lambda$

$C p$

$\chi$

$\mu$

$H_{L I}$
SI unit

$\mathrm{kg} \mathrm{m}^{-3}$

$\mathrm{J} \mathrm{m}^{-1} \mathrm{~s}^{-1} \mathrm{~K}^{-1}$

$\mathrm{J} \mathrm{kg}^{-1} \mathrm{~K}^{-1}$

$\mathrm{m}^{2} \mathrm{~s}^{-1}$

$\mathrm{kg} \mathrm{m}^{-1} \mathrm{~s}^{-1}$

$\mathrm{J} \mathrm{kg}^{-1}$
Water

999.8

0.561

4218

$1.3303 \times 10^{-7}$

0.001792

$3.334 \times 10^{5}$
Glaze ice

920

2.24

2092.7

$1.17 \times 10^{-6}$

$-$

TABLE 1. Physical properties of water and ice at $273 \mathrm{~K}$.

and thus these nonlinear terms could be neglected. For the typical case studied here, if $h_{0} \sim 3 \times 10^{-3} \mathrm{~m}, \beta \sim 10^{-2}$ and $\Delta T \sim 10 \mathrm{~K}$ are selected, and by using the physical properties of water and ice at $273 \mathrm{~K}$ shown in table 1 , then we have $R e \sim 10^{3}$, $S t \sim 10^{-2}, \Lambda_{1} \sim 10^{-6}, \Lambda_{2} \sim 10^{-5}$ and $\operatorname{Pr} \operatorname{Re} / S t \sim 10^{6}$, so the above conditions (2.9) are satisfied very well. In the following section, the considered basic flow is a Nusselt laminar flow without the motion of the ice-water interface, just like the water film falling along a solid plate. Then, even if the nonlinear terms are not neglected in advance, they will also be regarded as higher-order small terms and will not appear in the linearization equations.

\subsection{Linear stability equations}

The base steady-state solution (2.1) independent of the $x$-coordinate is given by a half-parabolic streamwise velocity profile, linear pressure distribution in the water and linear temperature distributions in both liquid and solid phases, which can be written as

$$
\begin{array}{ll}
\text { velocity: } & U(y)=1-y^{2}, \quad V(y)=0, \\
\text { pressure: } & P(y)=2 y \cot \beta, \\
\text { temperature: } & \Theta_{L}(y)=1-y, \quad \Theta_{I}(y)=T_{W}(y-1) /\left(H_{0}-1\right),
\end{array}
$$

where $y=H_{0}$ gives the coordinate of the cold wall. From the energy flux balance (2.6b) written at the ice surface for the unperturbed basic flow, it follows that $T_{W}=\left(1-H_{0}\right) / \lambda_{I L}$. Around the basic state, the disturbed flow can be decomposed as $u=U+u^{\prime}, v=v^{\prime}, p=P+p^{\prime}, \theta_{L}=\Theta_{L}+\theta_{L}^{\prime}, \theta_{I}=\Theta_{I}+\theta_{I}^{\prime}, \xi=\xi^{\prime}$ and $\eta=1+\eta^{\prime}$, then we can obtain the linearized perturbation equations and linearized boundary conditions. We expand the two-dimensional infinitesimal perturbations in the form of normal modes as

$$
\left(u^{\prime}, v^{\prime}, p^{\prime}, \theta_{L}^{\prime}, \theta_{I}^{\prime}, \xi^{\prime}, \eta^{\prime}\right)=\left[\hat{u}(z), \hat{v}(z), \hat{p}(z), \hat{\theta}_{L}(z), \hat{\theta}_{I}(z), \hat{\xi}, \hat{\eta}\right] \mathrm{e}^{\mathrm{i}(k x-\omega t)},
$$

where $k$ is the complex wavenumber in the $x$ direction, $\omega$ is the complex frequency. Substituting the normal modes into the linearized perturbation equations and linearized boundary conditions, and eliminating the eigenfunctions $\hat{u}$ and $\hat{p}$, we can obtain the linear instability equations

$$
\begin{gathered}
\left(\mathrm{D}^{2}-k^{2}\right)^{2} \hat{v}=\mathrm{i} \operatorname{Re}\left[(U k-\omega)\left(\mathrm{D}^{2}-k^{2}\right)-\mathrm{D}^{2} U k\right] \hat{v}, \\
\operatorname{Pr} \operatorname{Re}\left[\mathrm{i}(U k-\omega) \hat{\theta}_{L}-\hat{v}\right]=\left(\mathrm{D}^{2}-k^{2}\right) \hat{\theta}_{L} \\
-\mathrm{i} \omega \operatorname{Pr} \operatorname{Re} \hat{\theta}_{I}=\chi_{I L}\left(\mathrm{D}^{2}-k^{2}\right) \hat{\theta}_{I}
\end{gathered}
$$


and linear boundary conditions

$$
\begin{gathered}
\hat{\xi}=-\frac{\mathrm{i}}{U k-\omega} \hat{v}(0), \\
\left(\mathrm{D}^{2}+k^{2}\right) \hat{v}(0)-\frac{k}{U k-\omega} \mathrm{D}^{2} U \hat{v}(0)=0,
\end{gathered}
$$

$\left[\mathrm{D}^{2}-3 k^{2}-\mathrm{i} R e(U k-\omega)\right] \mathrm{D} \hat{v}(0)$

$$
\begin{gathered}
-\left[k^{2} R e^{-2 / 3}\left(\frac{3}{2} \sin \beta\right)^{-1 / 3} \zeta+2 \cot \beta\right] \frac{\mathrm{i} k^{2}}{U k-\omega} \hat{v}(0)=0 \\
\hat{\theta}_{L}(0)-\frac{\mathrm{i}}{U k-\omega} \mathrm{D} \Theta_{L} \hat{v}(0)=0 \\
\hat{\eta}=\frac{\mathrm{i}}{\phi_{I L} \omega} \hat{v}(1) \\
\mathrm{D} \hat{v}(1)+\frac{k \mathrm{D} U(1)}{\phi_{I L} \omega} \hat{v}(1)=0 \\
\lambda_{I L} \mathrm{D} \hat{\theta}_{I}(1)-\mathrm{D} \hat{\theta}_{L}(1)=\frac{\operatorname{Pr} R e}{S t} \hat{v}(1) \\
\hat{\theta}_{L}(1)=-\frac{\mathrm{i}}{\phi_{I L} \omega} \mathrm{D} \Theta_{L} \hat{v}(1) \\
\hat{\theta}_{I}(1)=-\frac{\mathrm{i}}{\phi_{I L} \omega} \mathrm{D} \Theta_{I} \hat{v}(1) \\
\hat{\theta}_{I}\left(H_{0}\right)=0
\end{gathered}
$$

Here, $\mathrm{D}=\mathrm{d} / \mathrm{d} y$. According to the media properties of the water and glaze ice at $273 \mathrm{~K}$ summarized in table 1 , we use Prandtl number $\operatorname{Pr}=13.47$, the ratio of thermal conductivities $\lambda_{I L} \approx 4.0$, the ratio of thermal diffusivities $\chi_{I L} \approx 0.1137$ and $\phi_{I L} \approx 0.0798$ in this paper.

\subsection{Numerical method and validations}

The linear stability equations are ordinary differential equations in terms of $\hat{v}, \hat{\theta}_{L}$ and $\hat{\theta}_{I}$, and can be regarded as a two-point boundary value problem. If there exists a nontrivial solution for the equations, a corresponding dispersion relation

$$
\mathrm{D}\left(k, \omega ; R e, S t, \zeta, H_{0}, \beta ; \operatorname{Pr}, \lambda_{I L}, \chi_{I L}, \phi_{I L}\right)=0
$$

should be satisfied, and we need to solve an eigenvalue problem. Because it is impossible to find the explicit analytical dispersion relation if there is no further simplification, the dispersion relation has to be obtained numerically. A compact fourth-order accurate finite-difference scheme is used to discretize the linear stability equations and boundary conditions by Shapiro \& Timoshin (2006) for this problem. In this paper, the Chebyshev collocation method (Canuto et al. 1988) is used to discretize the eigenvalue problem and the QZ algorithm to solve the resulting general eigenvalue problem. By using our spectral code, the surface mode, shear mode and ice mode of instability for small inclined angles are found, and the same neutral curves as the figure 5( $a$ ) in Shapiro \& Timoshin (2006) are reproduced within $1 \%$ precision. Furthermore, a detailed quantitative comparison of the critical parameters of the ice 


\begin{tabular}{lccccc} 
& \multicolumn{2}{c}{ Hu et al. } & & \multicolumn{2}{c}{ Shapiro \& Timoshin } \\
\cline { 2 - 3 } \cline { 5 - 5 } & $\zeta=0$ & $\zeta=4899.38$ & & $\zeta=0$ & $\zeta=4899.38$ \\
$\operatorname{Re}_{c}$ & 2866.5 & 3032.0 & & 2884.7 & 3041.4 \\
$k_{c}$ & 0.775 & 0.798 & & 0.772 & 0.796 \\
$c_{r c}$ & $-7.87 \times 10^{-5}$ & $-7.49 \times 10^{-5}$ & & $-7.93 \times 10^{-5}$ & $-7.53 \times 10^{-5}$
\end{tabular}

TABLE 2. Comparisons between Shapiro \& Timoshin (2006)'s results and ours on the critical parameters of the ice mode. St $=0.0625, H_{0}=10, \beta=0.002$.

mode is listed in table 2. Fine agreeable values are obtained from our code for critical Reynolds number, critical wavenumber as well as critical phase velocity. In $\S 3$, the linear absolute and convective instabilities of the ice mode are studied in detail with our validated spectral code.

\section{Linear absolute and convective instabilities}

Before we consider the spatio-temporal instability of the ice growth under the falling water film flows along an inclined ice plane, let us recall the basic concepts of the absolute/convective instability theory (AI/CI), which originated from plasma physics (Briggs 1964; Bers 1973). A good introduction to this theory is given by Huerre \& Monkewitz (1985, 1990), who first applied such a spatio-temporal stability analysis to spatially developing flows. They analytically investigated the absolute/convective nature of the instability through the asymptotic response of Green's function at large time. Furthermore, they indicated that periodic forcing on the boundary can be spatially amplified only when the system is convectively unstable.

Basically, when an amplifying wavepacket is convected away from its local position, the wavepacket would be said to be convectively unstable. If, otherwise, the amplification can be observed locally, the wavepacket would be said to be absolutely unstable. Generally, the absolute/convective nature of the instability is determined by the sign of the absolute growth rate $\omega_{0 i}=\operatorname{Im}\left[\omega\left(k_{0}\right)\right]$ defined at the saddle point $k_{0}$ of the dispersion relation, i.e. when $\left.(\mathrm{d} \omega / \mathrm{d} k)\right|_{k_{0}}=0$. In these expressions, $k$ is a complex wavenumber and $\omega$ is a complex frequency. If the absolute growth rate $\omega_{0 i}$ is greater than zero (lower than zero), the flow is said to be absolutely (convectively) unstable, and the boundary between absolute and convective instabilities is then determined by a zero absolute growth rate, i.e. $\omega_{0 i}$. However, note that the saddle point $k_{0}$ used to identify AI/CI must satisfy the Briggs-Bers collision criterion, i.e. the saddle point must be a pinch point produced by two distinct spatial branches of solutions of the dispersion relation, $k_{n}^{ \pm}(\omega)$, coming, respectively, from the upper and lower half $k$ planes and commonly referred to as the upstream and downstream branches. In this paper, the saddle points have all been found to satisfy the collision criterion.

In order to clearly investigate the spatio-temporal evolutionary characteristics of the unstable flow, it is necessary to study the response of the flow to a localized disturbance along an arbitrary fixed spatio-temporal ray, $V=x / t$, as $t \rightarrow \infty$. This is equivalent to analysing how the response evolves in a reference frame moving at the velocity $V$. Upon introducing the Doppler-shifted frequency $\omega^{v}=\omega-V k$ and $k^{v}=k$, the dispersion relation in the moving coordinate system would be

$$
\mathrm{D}_{v}\left(k^{v}, \omega^{v}\right)=\mathrm{D}\left(k^{v}, \omega^{v}+V k^{v}\right)=0 .
$$



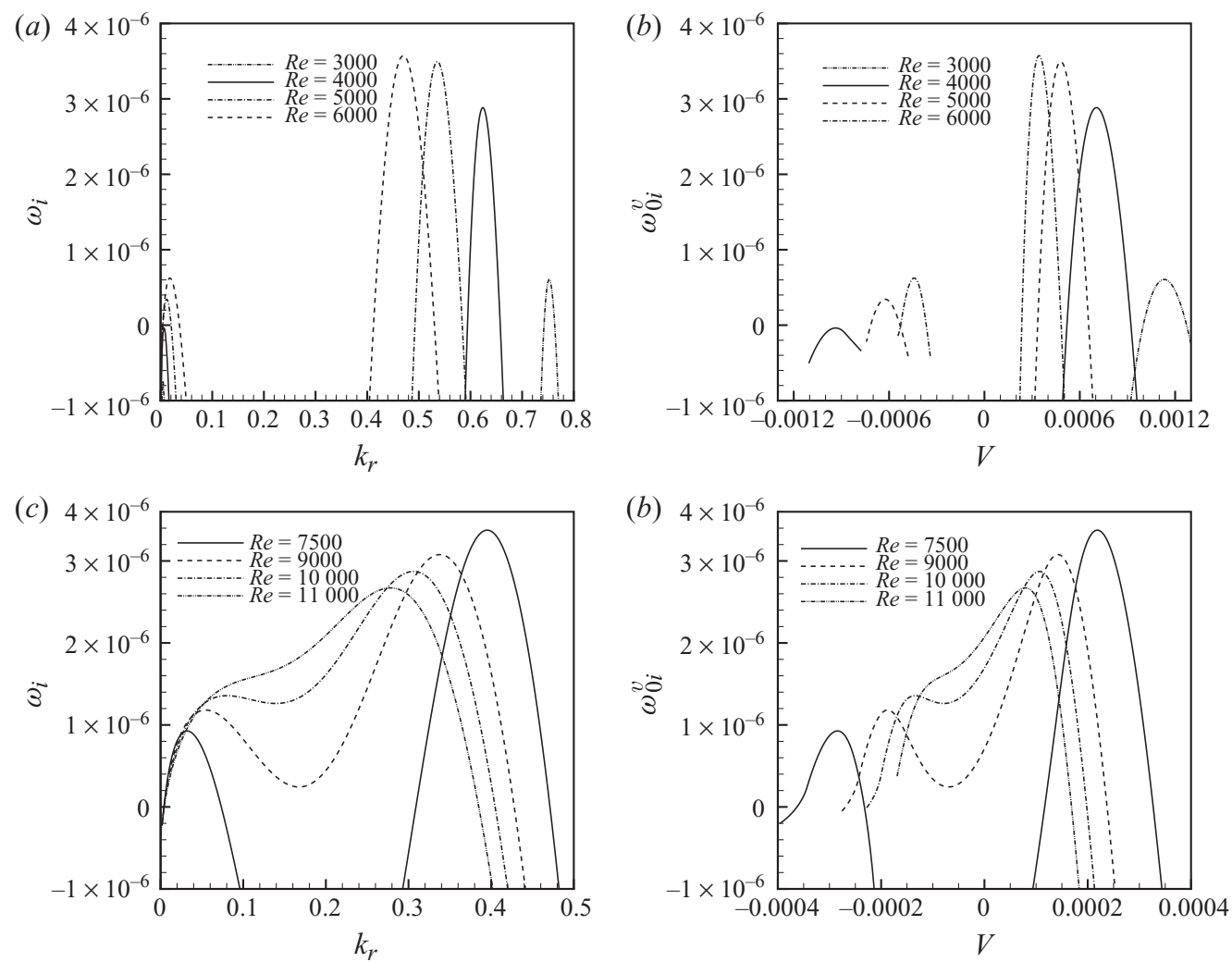

FIgURE 2. $(a, c)$ Temporal growth rates $\omega_{i}$ of the ice mode as a function of real wavenumber $k_{r}$ and $(b, d)$ local spatio-temporal growth rates $\omega_{0 i}^{v}$ of the ice mode as a function of the ray velocity $V$ for different Reynolds numbers. $S t=0.0625, \zeta=0, H_{0}=10, \beta=0.002$.

Because of $\mathrm{d} \omega^{v} / \mathrm{d} k^{v}=0$, the saddle point $\tilde{k}$ will take place at

$$
\mathrm{D}(\tilde{k}, \tilde{\omega})=0 \quad \text { and } \quad \frac{\mathrm{d} \omega}{\mathrm{d} k}(\tilde{k}, \tilde{\omega})=V
$$

and then the absolute growth rate in the moving frame is obtained at $k_{0}^{v}=\tilde{k}$ through

$$
\omega_{0}^{v}=\tilde{\omega}-V \tilde{k}
$$

Here also, the saddle point $\tilde{k}$ obtained from (3.2) must verify the Briggs-Bers collision criterion mentioned above. The saddle points in this paper will be obtained numerically by Newton-type iterations (Deissler 1987; Yin et al. 2000).

First, for the unstable ice mode of the flow system, the temporal growth rates $\omega_{i}$ versus real wavenumber $k_{r}$ and local spatio-temporal growth rates $\omega_{0 i}^{v}$ as a function of observer velocity $V$ for different Reynolds numbers are plotted in figure 2. As is well known, the maximum growth rates for the temporal instability and spatiotemporal instability are equivalent for the zero imaginary part of the saddle point occurs at that point. Thus, it is clearly seen from figures $2(a)$ and $2(b)$, there exist two separate unstable branches in the flow system: one is long-wave unstable and the long-wave disturbance convects upstream (upstream branch), the other branch has finite wavelength and its unstable wavepacket propagates downstream (downstream branch). With increasing the Reynolds number from $R e=3000$ to $R e=6000$, the 
maximum growth rates increase for the upstream and downstream branches, and the flows are convectively unstable because the amplification of disturbance cannot be obtained locally in the laboratory framework, i.e. the absolute growth rate $\omega_{0 i}$ is less than zero. In figure 2(a), the temporal unstable region of the real wavenumber $k_{r}$ for both upstream and downstream branches becomes large, while the unstable wavenumber increases for the upstream branch and decreases for the downstream branch. Accordingly in figure $2(b)$, the spatio-temporal unstable region of the convective velocity $V$ for both upstream and downstream branches becomes small, while the convective velocity increases for the upstream branch and decreases for the downstream branch. Thus, from both temporal and spatio-temporal point of view, the two branches become more unstable and move closer. For $R e=4000$, the downstream branch is already unstable even for $R e=3000$, while the upstream branch is stable now. The two branches will determine two critical curves such as the $R e-\beta$ critical curves shown later. Furthermore, across the critical curves, there exist not only the critical wavenumber $k_{c}$ for the temporal instability but also the critical convective velocity $V_{c}$ for the spatio-temporal instability.

Furthermore when increasing the Reynolds number from $R e=7500$ to $R e=11000$, as shown in figures $2(c)$ and $2(d)$, the unstable regions of the real wavenumber and the observer velocity for the two branches become more closer and have already connected into one branch at $R e=9000$, which produces a local minimum where the growth rate is greater than zero. Also, it is easily seen from figure $2(d)$ that the absolute growth rate is greater than zero for $R e=9000$, then the flow system becomes absolutely unstable. When $R e=10000$, the upstream maximum growth rate further increases as well as the local minimum, while the downstream maximum growth rate decreases. When $R e=11000$, the upstream maximum growth rate and the local minimum growth rate disappear, thus the connected branch has only one maximum growth rate which corresponds to the origin downstream branch.

Secondly, because of the existence of the transition between the linear absolute and convective instability, it is important to plot the AI/CI boundary curve in the parameter region. As shown in figure 3, the AI/CI boundary curve and critical Reynolds number curves for the downstream and upstream branches are plotted in the $R e-\beta$ parameter region. It is found that with increasing the inclined angle from 0.004 , the Reynolds number on the AI/CI boundary curve decreases and arrives at a minimum value where the inclined angle is about 0.0208 ; then the AI/CI transition Reynolds number increases slowly with the increase of the inclined angle, or in other words with the increase of the Reynolds number, the inclined angle on the $\mathrm{AI} / \mathrm{CI}$ boundary curve increases and quickly arrives at a maximum value where the Reynolds number is about 814 ; finally, across that maximum point the AI/CI transition-inclined angle decreases when increasing the Reynolds number. From the AI/CI boundary curve, it is easily seen that when the Reynolds number is smaller than the minimum value or the inclined angle is larger than the maximum value, the absolute instability does not exist.

Beyond the absolute instability region, the critical curve for the downstream branch (dashed line) intersects with the upstream branch (dash-dotted line) and the AI/CI boundary curve (solid line). Note that the critical curve for the downstream branch disappears near $R e=829.8$ and $\beta=0.01345$ because the local maximum growth rate for the branch does not already exist, and a very small piece of the critical curve is located at the absolute instability region though it is not obvious from figure 3 . The critical convective velocities $V_{c}$ on the two critical curves are always positive and negative for the downstream and upstream branch, as shown in figure 4 . It is 


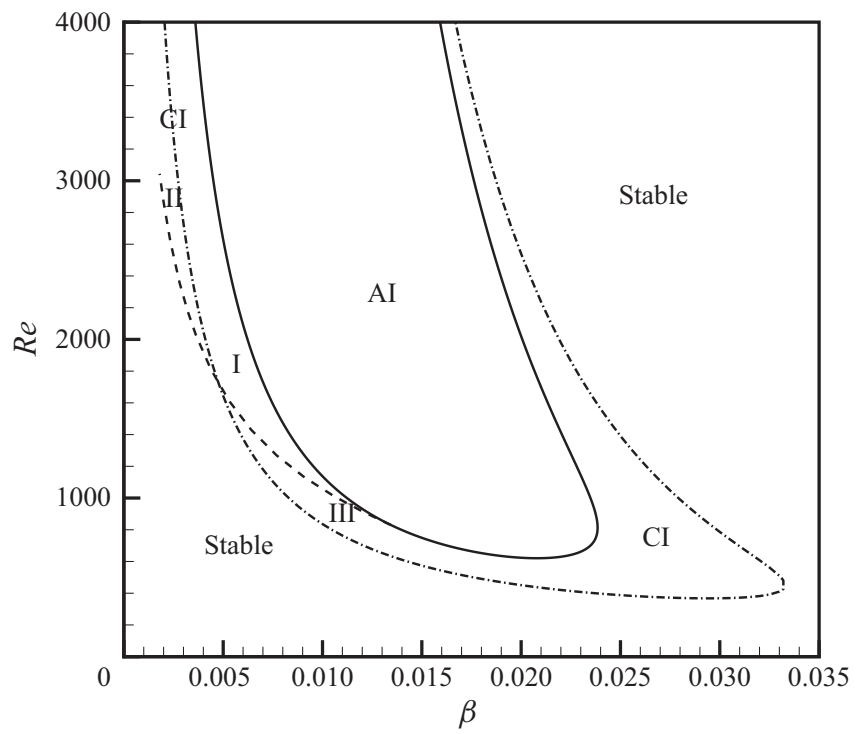

FIGURE 3. AI/CI boundary curve with solid line and critical Reynolds number curves with dashed line for the downstream branch and the dash-dotted line for the upstream branch as a function of the inclined angle. $S t=0.0625, \zeta=0, H_{0}=10$.

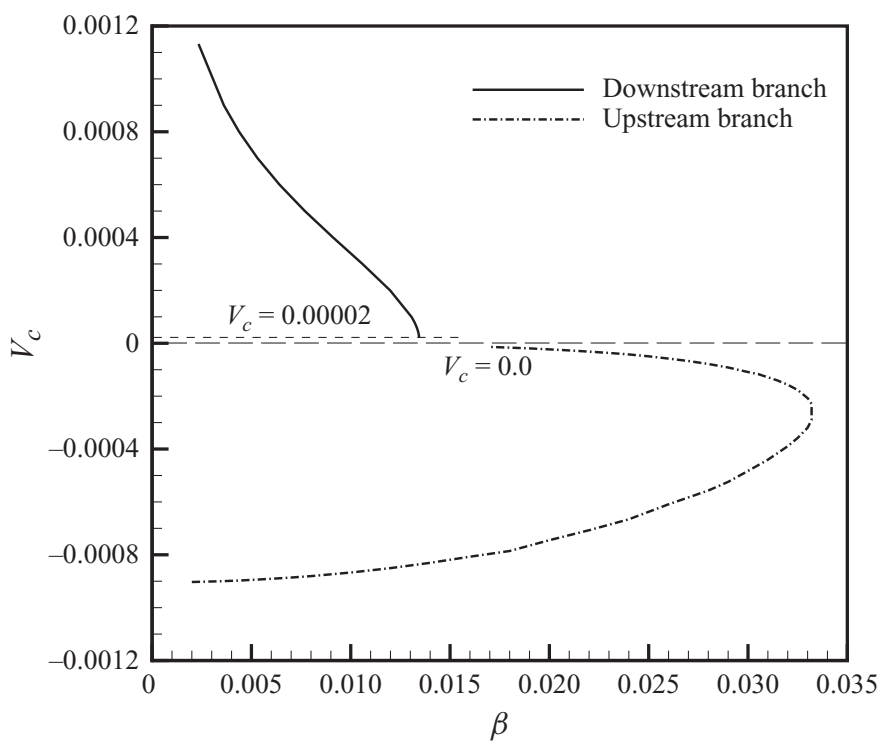

FIGURE 4. Critical convective velocities $V_{c}$ on the critical curves of the downstream (solid line) and upstream (dash-dotted) branches as a function of the inclined angle. $S t=0.0625, \zeta=0$, $H_{0}=10$.

found that for the downstream branch the critical convective velocity disappears near $V_{c}=0.00002$, while the critical convective velocity of the upstream branch is always below $V_{c}=0.0$ and approaches to it with decreasing the inclined angle. Thus, the intersection of the two critical curves divides the convective unstable region into three parts: parts I, II and III. These parts have different features as follows. 


$\begin{array}{lcc}\text { Part } & \beta & R e \\ \text { I } & 0.005 & 2000 \\ \text { II } & 0.003 & 2400 \\ \text { III } & 0.009 & 1000\end{array}$

TABLE 3. Selected points in the convectively unstable region. $S t=0.0625, \zeta=0, H_{0}=10$.
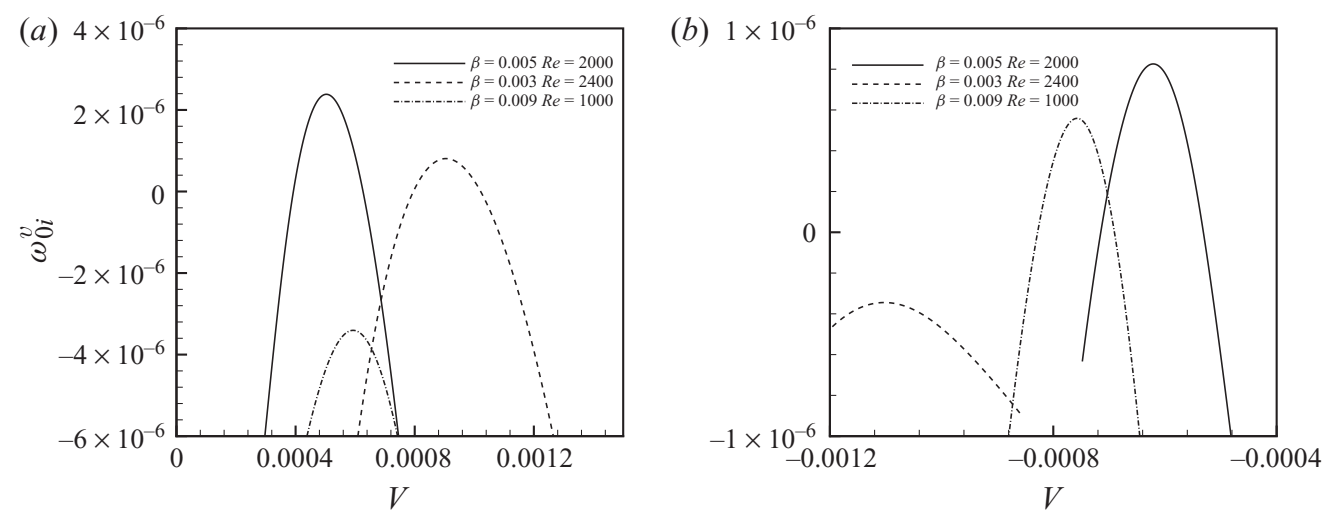

FiguRE 5. Spatio-temporal growth rates $\omega_{0 i}^{v}$ as a function of the $(a)$ positive and $(b)$ negative ray velocities $V$ at different inclined angles and Reynolds numbers. $S t=0.0625, \zeta=0$, $H_{0}=10$.

(i) Part I: under local disturbance of the basic flow, there exist the two convectively unstable wavepackets. One propagates downstream because of the downstream unstable branch and the other upstream due to the upstream unstable branch.

(ii) Part II: there exists one convectively unstable wavepacket that propagates only downstream because of the downstream unstable branch and upstream stable branch.

(iii) Part III: there exists one convectively unstable wavepacket that propagates only upstream because of the upstream unstable branch and the downstream stable branch or because of the non-existence of the downstream branch for larger inclined angles $(\beta>0.01345)$.

In order to exhibit the above different convectively unstable characteristics for the three parts, a group of parameters is selected for the inclined angle and Reynolds number, as shown in table 3, to plot local growth rates $\omega_{0 i}^{v}$ as a function of observer velocity $V$, as shown in figure 5 . These two figures clearly show that the flow with $\beta=0.005$ and $R e=2000$ in part I has both downstream and upstream convectively unstable wavepackets; while the flow with $\beta=0.003$ and $R e=2400$ in part II has only downstream convectively unstable wavepacket, and the flow with $\beta=0.009$ and $R e=1000$ in part III has only upstream convectively unstable wavepacket.

Finally, the AI/CI boundary curves in the parameter $R e-\beta$ region for different Stefan numbers and different thicknesses of the ice layer are plotted in figure 6 . It is clearly seen from figure 6 $(a)$ that with decreasing the Stefan number from $S t=0.0625$ to $S t=0.0025$, the absolute instability region becomes larger and occurs at much smaller Reynolds number and larger inclined angle. From figure 6(b), it is obvious that with increasing the thickness of the ice layer, the absolute instability region also increases from $H_{0}=5$ to $H_{0}=10$. However, for large thickness of the ice layer such 

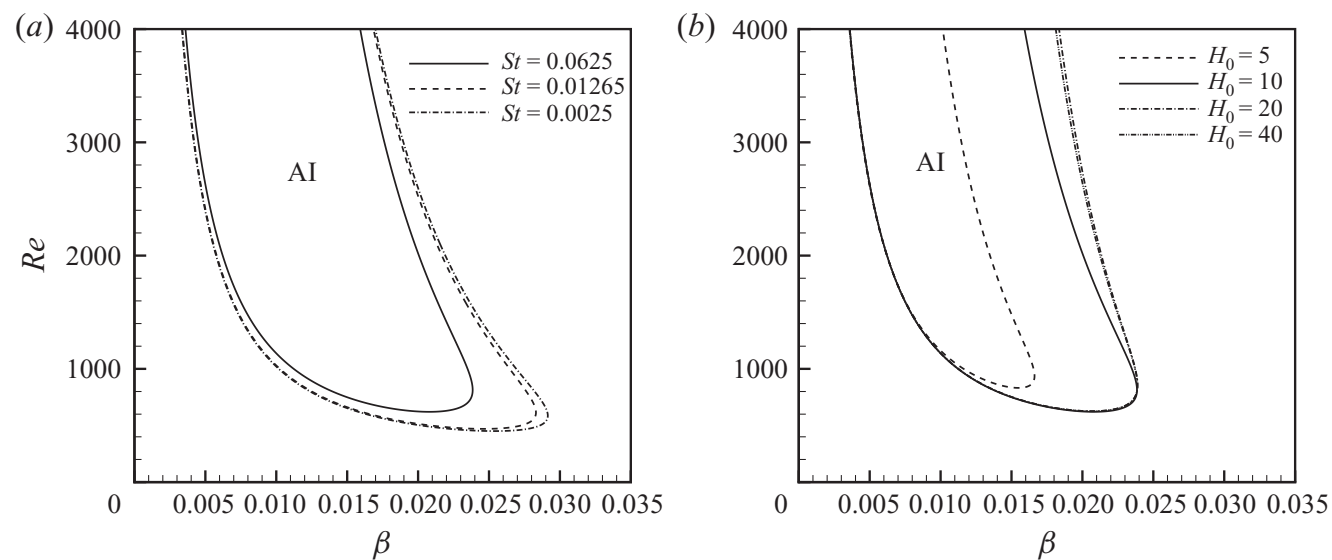

FIGURE 6. AI/CI boundary curves in the parameter $R e-\beta$ region for $(a)$ different Stefan numbers with $H_{0}=10$ and $(b)$ different thicknesses of the ice layer with $S t=0.0625$. $\zeta=0$.
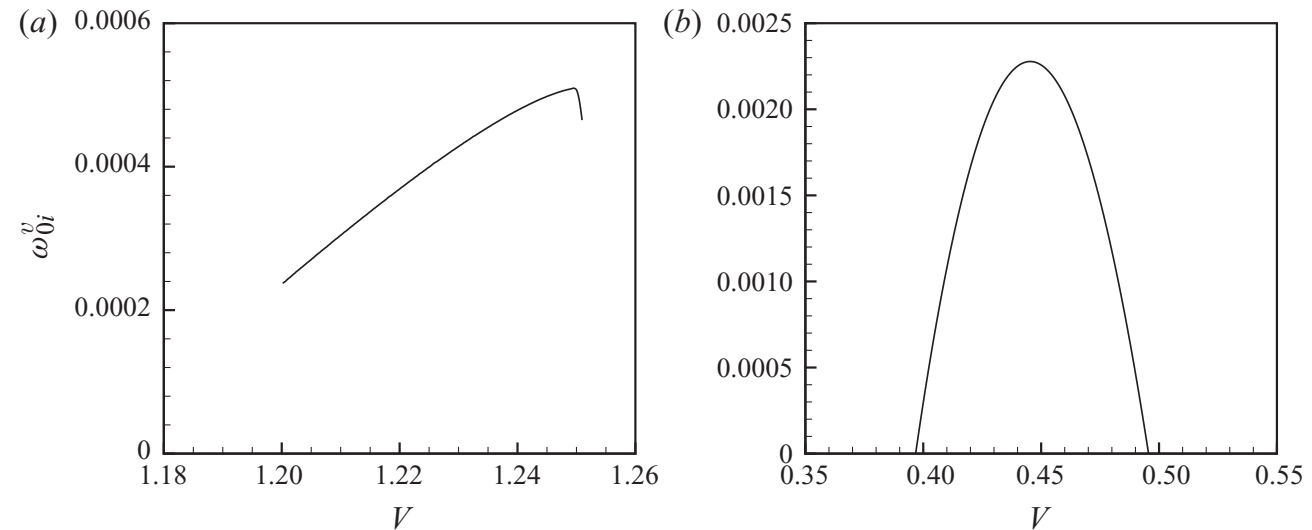

FIGURE 7. Spatio-temporal growth rates $\omega_{0 i}^{v}$ as a function of the ray velocity $V$ for $(a)$ the surface wave instability and $(b)$ the shear instability. $S t=0.0625, \zeta=0, H_{0}=10, \beta=0.002$, $R e=6000$.

as $H_{0}=20$ and $H_{0}=40$, the AI/CI boundary curves change little and the absolute instability region becomes a little smaller only.

Note that for the surface wave mode, the critical Reynolds number is approximately very well equal to $R e_{c}=5 / 4 \cot \beta$, which is the critical value for the isothermal situation. Thus, the critical boundary curve for the surface wave instability (for clarity, not plotted in figure 3) is below those for the ice mode in the $R e-\beta$ parameter region. Similarly, we have checked that the surface wave instability spreads convectively downstream with a speed higher by 3 orders and a spatio-temporal growth rate larger by 2 orders than the ice mode, as seen from figures 5 and $7(a)$. It is also obvious from figure $7(b)$ that the shear instability is convectively unstable and spreads downstream with a slower speed than the surface wave instability, but with a much larger spatiotemporal growth rate. It is also found that with the variation of the Stefan number and the thickness of the ice layer, the curves of spatio-temporal growth rates have no change, which shows that the ice-induced instability has little effect on the surface wave instability and the shear instability of the film flows. Because of the convectively 
unstable characteristics of the surface wave instability and the shear instability, the $\mathrm{AI} / \mathrm{CI}$ boundary curves shown in this section for the ice mode are just the AI/CI boundary curves for the whole system.

\section{Conclusions}

In this paper, a linear absolute and convective instability analysis of ice growth under a falling water film is performed for the ice mode through the computation of the normal modes of the full linearized stability equations with the Chebyshev collocation method. It is found that there exist downstream and upstream branches for the Reynolds number $R e=7500$, which is above the two critical Reynolds numbers with the inclined angle $\beta=0.002$. When increasing the Reynolds number to $R e=9000$, the two branches move closer and connect with each other, then absolute instability occurs. By plotting the AI/CI boundary curve and critical curves in the $R e-\beta$ parameter region, the flow system is divided into stable, convectively unstable and absolutely unstable regions. Furthermore, the downstream and upstream critical curves divide the convectively unstable region into three parts, where the flow maybe convectively both downstream and upstream unstable (part I) or may be only convectively unstable in only one direction (downstream for part II and upstream for part III). From these plotted curves, it is found that the (absolute) instability does not appear either below a minimum Reynolds number or above a maximum inclined angle.

We are grateful to the anonymous referees for suggestions that significantly improved the paper. This work is supported by the Joint Fund of NSAF (grants 10676005 and 10676004), the National Natural Science Foundation of China (NSFC; grants 10702011 and 10802086), the Science Foundation of CAEP (grant 2009B0201023) and the Scientific Research Foundation for the Returned Overseas Chinese Scholars, State Education Ministry.

\section{REFERENCES}

Benjamin, T. B. 1957 Wave formulation in laminar flow down an inclined plane. J. Fluid Mech. 2, 554-574.

Bers, A. 1973 Theory of absolute and convective instabilities. In International Congress on Waves and Instabilities in Plasma (ed. G. Auer \& F. Cap), pp. B1-B52. Institute for Theoretical Physics, Innsbruck.

Brevdo, L., Laure, P., Dias, F. \& Bridges, T. J. 1999 Linear pulse structure and signalling in a film flow on an inclined plane. J. Fluid Mech. 396, 37-71.

Briggs, R. J. 1964 Electron-Stream Interaction with Plasmas. MIT Press.

Canuto, C., Hussaini, M. Y., Quarteroni, A. \& Zang, T. A. 1988 Spectral Methods in Fluid Dynamics. Springer.

DeBruin, G. J. 1974 Stability of a layer of liquid flowing down an inclined plane. J. Engng Math. 8, 259-270.

DeISSLER, R. J. 1987 Spatially growing waves, intermittency, and convective chaos in an open-flow system. Physica D 25, 233-260.

Floryan, J. M., Davis, S. H. \& Kelly, R. E. 1987 Instability of a liquid film flowing down a slightly inclined plane. Phys. Fluids 30, 983-989.

GiLPIN, R. R. 1979 The morphology of ice structure in a pipe at or near transient Reynolds numbers. AIChE Symp. Ser. 75, 89-94.

Huerre, P. \& Monkewitz, P. A. 1985 Absolute and convective instabilities in free shear layers. J. Fluid Mech. 159, 151-168. 
Huerre, P. \& Monkewitz, P. A. 1990 Local and global instabilities in spatially developing flows. Annu. Rev. Fluid Mech. 22, 473-537.

KapitZA, P. L. \& KapitZA, S. P. 1949 Wave flow of thin layers of a viscous fluid. Zh. Eksper. Teor. Fiz. 19, 105-120, also in Collected Papers of P. L. Kapitza (ed. D. Ter Haar), pp. 690-709. Pergamon, 1965.

Lin, S. P. \& Hudman, M. 1996 Nonequilibrium evaporation from a heated liquid layer. $J$. Thermophys. Heat Transfer 10, 497-503.

Shapiro, E. 2004 Instabilities in multiphase and icing flows. PhD thesis, University of London, London.

Shapiro, E. \& Timoshin, S. 2006 Linear stability of ice growth under a gravity-driven water film. Phys. Fluids 18, 074106.

Shapiro, E. \& Timoshin, S. 2007 On ice-induced instability in free-surface flows. J. Fluid Mech. 577, $25-52$.

Woods, D. R. \& Lin, S. P. 1996 Critical angle of shear wave instability in a film. J. Appl. Mech. 63, 1051-1052.

YIH, C. S. 1963 Stability of liquid flow down an inclined plane. Phys. Fluids 6, 321-334.

YIN, X.-Y., Sun, D.-J., WeI, M.-J. \& Wu, J.-Z. 2000 Absolute and convective instability character of slender viscous vortices. Phys. Fluids 12, 1062-1072. 